\title{
Oxidation of Cyclopropane Terpenoids with Ruthenium Tetraoxide
}

\author{
Takahiro KawAI, Takashi OoI, and Takenori Kusumi* \\ Faculty of Pharmaceutical Sciences, Tokushima University; Tokushima 770-8505, Japan. \\ Received October 31, 2002; accepted December 18, 2002
}

\begin{abstract}
Oxidation products of cyclopropanoid terpenes, (-)-carane (4), (+)-cyclosativene (5), laurinterol methyl ether (6), and thujopsane (7), with ruthenium tetraoxide were investigated.
\end{abstract}

Key words absolute configuration; ruthenium tetraoxide; terpene; cyclopropane

Recently, we have reported the isolation of a new sesquiterpene, cyclosinularane (1) from a soft coral collected off Ishigaki Island. ${ }^{1)}$ The compound is a tetracyclic hydrocarbon composed of a cyclopropane ring. Its absolute configuration has been undetermined because it is an oily substance and no oxygen function necessary for chemical derivatization is present. If a carbonyl group could be introduced on one of the methylene carbons, the absolute configuration would be determined by means of the modified Mosher's method ${ }^{2)}$ after the carbonyl is reduced to a hydroxy group. This seemed feasible because Hasegawa and co-workers reported that ruthenium tetraoxide oxidation of cyclopropyl hydrocarbons afforded $\alpha$-ketocyclopropanes as exemplified by reaction 2 to $3 .{ }^{3)}$ Coudret and co-workers also reported the same category of the reactions. ${ }^{4)}$

Because of the limited amount of 1, (-)-carane (4), (+)cyclosativene (5), laurinterol methyl ether (6), and thujopsane (7) were chosen as substrates, and they were subjected to ruthenium tetraoxide oxidation.

\section{Results and Discussions}

The reaction conditions were the same as those reported by Hasegawa and co-workers. ${ }^{3)}$ The substrate was treated with ruthenium chloride $(0.2-0.3 \mathrm{eq})$ and an excess ( $3 \mathrm{eq})$ of sodium periodate in carbon tetrachloride-acetonitrile and a phosphate buffer solution ( $\mathrm{pH} 7)$. The reaction was carried out at room temperature (rt) for $24-26 \mathrm{~h}$.

Oxidation of (-)-Carane and Application of the Modified Mosher's Method (-)-Carane (4) was prepared from commercially available $(+)-3$-carene by borane reduction. The ruthenium oxide oxidation of 4 for $24 \mathrm{~h}$ afforded 5 -caranone (8) in $75 \%$ yield. The ketone was reduced with diisobutylaluminum hydride, and the volatile alcohol (9) was, without purification, converted to $(R)$ - and $(S)$-methoxy(phenyl)trifluoromethylacetic acid (MTPA) esters $(\mathbf{1 0}, \mathbf{1 1})$ by using 2,4,6-trinitrochlorobenzene as a condensation reagent. ${ }^{5}$ ) The $\beta$-configuration of the oxygen function at C-5 was determined by the nuclear Overhauser effects (NOEs) observed between $5 \alpha-\mathrm{H}$ and $3 \alpha-\mathrm{H}$ as well as $5 \alpha-\mathrm{H}$ and $6 \alpha-\mathrm{H}$ observed for 11. The $\Delta \delta$ values $\left[\delta_{(S) \text {-MTPA }}-\delta_{(R) \text {-MTPA }}\right]$ were calculated as shown in 12. ${ }^{2)}$ The absolute configuration predicted on the basis of these values is identical with the known one.

These results indicated that combination of the ruthenium tetroxide oxidation and the modified Mosher's method would be useful for the absolute configuration determination of cyclopropane terpenoids.

Encouraged by the positive findings, we further advanced toward more complex cyclopropanoids, which turned out to produce unexpected products.

Oxidation of (+)-Cyclosativene Commercially available $(+)$-cyclosativene $(\mathbf{5})$ afforded three products, 13 (15\%), $14(7 \%)$ and $16(5 \%)$, by the ruthenium oxidation.

The major product 13 exhibited a molecular ion at $\mathrm{m} / \mathrm{z}$ $220.1835\left(\mathrm{C}_{15} \mathrm{H}_{24} \mathrm{O}\right)$ and it showed a carbon signal at $\delta 74.5$ (s) due to an oxygenated quaternary carbon in its ${ }^{13} \mathrm{C}-\mathrm{NMR}$ spectrum. The cyclosativene framework and the position of the hydroxy group were deduced by the extensive study of the two dimensional (2D) NMR spectra including the heteronuclear multiple bond connectivity (HMBC) spectrum. The correlations observed between protons and carbons are illustrated in 13a. The orientation of the hydroxy group was supposed to be $\alpha$, that is C-5(R), by the cross peaks observed in the nuclear Overhauser effect spectroscopy (NOESY) spectrum [13b], and the $\alpha$-orientation was further confirmed by the titration study using a paramagnetic shift reagent, $\left.\mathrm{Eu}(\mathrm{fod})_{3}{ }^{6}{ }^{6}\right)$ The downfield shift of $\mathrm{H}-1$ is greater $(\Delta \delta=0.46)$ than that of $\mathrm{H}-7(\Delta \delta=0.25)$ when $\mathrm{Eu}(\mathrm{fod})_{3} / \mathbf{1 3}=0.27$ in 54 $\mathrm{mM} \mathrm{CDCl}_{3}$ solution of 13 .

The second product had an unexpected structure. The molecular formula was established by high resolution (HR)-MS as $\mathrm{C}_{14} \mathrm{H}_{21} \mathrm{ClO}$, showing that one carbon was eliminated from cyclosativene (5) and a chlorine atom was incorporated, which was verified by appearance of $\mathrm{M}+2$ ion at $\mathrm{m} / \mathrm{z} 242$ (33\% intensity of the molecular ion). The IR absorption at $1748 \mathrm{~cm}^{-1}$ and the ${ }^{13} \mathrm{C}$ signal at $\delta 216.8$ indicated the presence of a cyclopentanone moiety. Two possible structures, 14 and 14', were deducible for this product even after the 2D NMR (heteronuclear single quantum coherence (HSQC), HMBC, NOESY etc.) studies, because there was an ambiguity in the assignment of two methine signals at $\delta_{\mathrm{C}} 57.1 / \delta_{\mathrm{H}}$ 3.68 (brs) and $\delta_{\mathrm{C}} 61.4 / \delta_{\mathrm{H}} 2.77$ (br s). These signals could be assigned as C-7 and C-9 of either 14 or $14^{\prime}$. Therefore, this product was transformed into an alcohol (15) by reduction with sodium borohydride. C-8 of 15 appears at $\delta 80.6$, and by the HSQC spectrum, H-8 ( $\delta 3.81)$ was assignable. H-8 is coupled with H-9 $(\delta 2.47)(J=3.6 \mathrm{~Hz})$ which was further correlated with a methine $\left(\delta_{\mathrm{H}} 4.25 / \delta_{\mathrm{C}} 56.5\right)$ assignable as H-7. Structure 15 was eventually confirmed by the NOESY spectrum (see 15), and these findings led to the structure of 14 as the oxidation product.

The third oxidation product $\left(\mathrm{C}_{15} \mathrm{H}_{22} \mathrm{O}_{4}\right.$ by HR-MS: $\mathrm{m} / \mathrm{z}$ $267.1597[\mathrm{M}+1])$ showed two signals of ester carbonyl carbons at $\delta 167.1(\mathrm{~s})$ and $168.0(\mathrm{~s})$. The IR spectrum exhibited a strong absorption band at $1749 \mathrm{~cm}^{-1}$, suggesting the presence of strained ester moieties. In the ${ }^{13} \mathrm{C}-\mathrm{NMR}$ spectrum an acetalic carbon shows a signal at $\delta 110.1$ (C-8), to which two 


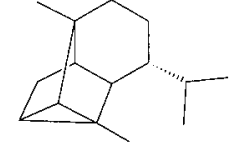

1 (cyclosinularane)
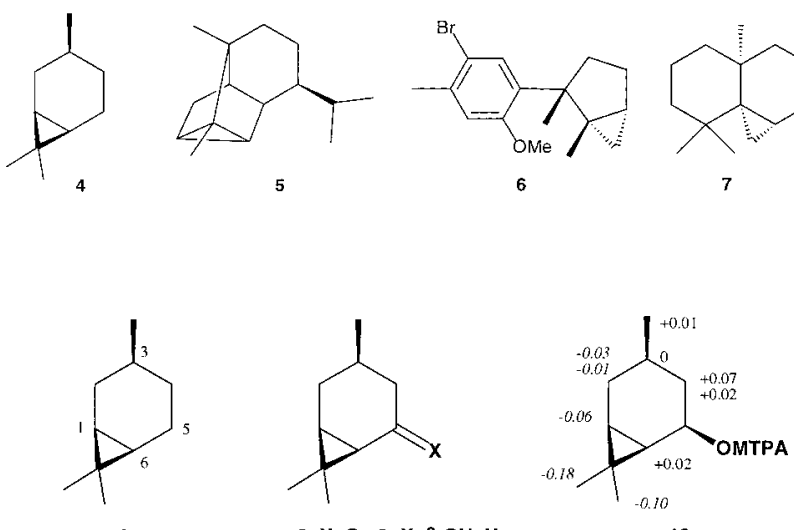

12<smiles>CC1CCC2C(C1)C2(C)C</smiles>

$\begin{aligned} \text { 8: } X & =O \quad 9: X=\beta-O H, H \\ 10: X & =\beta-(R)-M T P A-O, H\end{aligned}$ 11: $X=\beta-(S)-M T P A-O, H$
$0 .-M T P A$

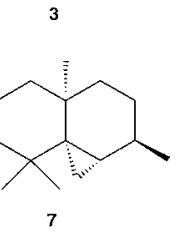

Fig. 1. A Possible mechanism to Produce the Chloroketone (14) from Cyclosativene (5)

Here we considered the pathway to produce 13, 14, and 16. Inertness of the methylene group at C-10 of cyclosativene (5) against oxidation may be ascribed to the steric hindrance by the surrounding aliphatic moieties, especially from methyl-11. It has been reported that a hydroxy group is introduced to a tertiary carbon, ${ }^{7)}$ as in the case of 13 , by oxidation with ruthenium tetraoxide, which can oxidize a methine rather than a methylene or methyl because the $\mathrm{C}-\mathrm{H}$ bond of the former is more electron-rich than that of the latter. The oxidation at C-5 took place from the less crowded side to give the $\alpha$-hydroxy product (13).

Formation of the chloroketone (14) is quite puzzling. The chlorine atom apparently originated from ruthenium chloride. A possible pathway leading to $\mathbf{1 4}$ is described in Fig. 1. Although disfavored, the methyl hydrogens can be nevertheless attacked by $\mathrm{RuO}_{4}{ }^{8}{ }^{8}$ Then a chloride anion attacks at $\mathrm{C}-7$ carbon with concomitant $\mathrm{C} 7-\mathrm{C} 8$ bond cleavage. Oxidative cleavage of the exomethylene group with ruthenium tetraoxide affords 14. It is peculiar, however, that the chlorine atom is introduced from the more hindered side (C-7) rather than from the less crowded direction (C-9).

Peroxidation of the cyclopropane ring of 5, two methines at $\mathrm{C}-7$ and 9 giving carboxyl groups and the quaternary carbon at C-8 transforming to a ketone $\left(\mathbf{1 6}^{\prime}\right)$, produces $\mathbf{1 6}$. To our best knowledge, this type of oxidation of 1,1,2,3-tetrasubstituted cyclopropane may be the unprecedented one.

Oxidation of Laurinterol Methyl Ether Laurinterol was obtained from the red alga Laurencia intermedia YAMADA. ${ }^{9)}$ The phenolic hydroxy group was converted into a methyl ether (6) to prevent the oxidation of the phenyl group. Oxidation of $\mathbf{6}$ under the conditions described above afforded a product $\left(\mathrm{C}_{16} \mathrm{H}_{19} \mathrm{BrO}_{2}\right.$ : HR-MS $m / z$ 322.0546) in $32 \%$ yield. This compound showed an intense IR absorption band at $1660 \mathrm{~cm}^{-1}$ due to a conjugated enone. Presence of the enone system was further confirmed by the singlets at $\delta 5.88(1 \mathrm{H})$ and $1.65(3 \mathrm{H})$ ascribable to $\alpha$-proton of the $\alpha, \beta$-unsaturated ketone and the olefinic methyl, respectively, in the ${ }^{1} \mathrm{H}-\mathrm{NMR}$ spectrum and by the olefinic carbon signals at $\delta 169.4$ (s) and 125.8 (d) and a carbonyl carbon signal at $\delta 198.9$ in the ${ }^{13}$ C-NMR spectrum. Presence of 5-bromo-2-methoxy-4methylphenyl group and vicinal two methylene groups were obvious from the NMR properties, and structure 18 was assigned for this oxidation product.

Instead of oxidizing the methylene group (C-5) adjacent to the cyclopropyl moiety, ruthenium tetraoxide cleaved, as in the case of 14, one (C2-C4) of the cyclopropyl bonds to give possibly the hydroxy ketone (17), which afforded 18 by dehydration. methyl signals at $\delta 1.59(\mathrm{~s})(\mathrm{H}-15)$ and $1.17(\mathrm{~s})(\mathrm{H}-11)$ showed correlation peaks in the HMBC spectrum. On the basis of these properties as well as other NMR data, structure 16 was assigned to this product. 
Oxidation of Thujopsane We were further interested in the mode of ruthenium tetraoxide oxidation of a cyclopropane compound. Thujopsane (7), obtained by reduction of thujopsene with borane, was chosen as another example and subjected to the ruthenium oxidation.

The product turned out to be a ketocarboxylic acid (19) that was isolated as a methyl ester (20) after treatment with diazomethane. It should be remarkable that the same compound (19) was obtained by oxidation of thujopsene under the same conditions. Although the latter type of oxidative cleavage of an olefin is already documented, ${ }^{10,11)}$ the rapture of the sigma bond adjacent to a cyclopropane ring into a dicarbonyl moiety must be unique.

Coudret and co-workers reported the same type of oxidative cleavage in the ruthenium oxide oxidation of cyclopropane compounds. $\left.{ }^{4}\right)$ According to the proposed mechanism, a hydroxy group is at first introduced at C-2 as in the case of 13, the tertiary alcohol is dehydrate to thujopsene, and then the oxidative cleavage of the double bond takes place.

\section{Conclusion}

Four cyclopropane terpenoids, (-)-carane $(4),(+)$-cyclosativene (5), laurinterol methyl ether (6), and thujopsane (7) were subjected to ruthenium tetraoxide oxidation. (-)Carane (4) afforded a ketone (8), the reduction product (9) of which was subjected to the modified Mosher's method to confirm the absolute configuration. In the case of $\mathbf{5}$ and $\mathbf{6}$, carbonyl functions were not introduced at the $\alpha$-methylenes of the cyclopropane rings. (+)-Cyclosativene (5) afforded a tertiary alcohol $\mathbf{1 3}$ as well as a chloroketone $\mathbf{1 4}$ and an acetal diester 16, which resulted from the oxidative degradation of the cyclopropane ring. The cyclopropane ring of laurinterol methyl ether (6) was labile under the oxidation conditions, and the reaction produced a cyclohexenone (18). The ruthenium oxidation of thujopsane (7) resulted in the unusual cleavage of the $s p^{3}-s p^{3}$ sigma bond $\beta$ to the cyclopropane ring to produce a ketocarboxylic acid (19).

The result obtained for (+)-cyclosativene (5) is informative for elucidating the absolute configuration of cyclosinularane (1): Formation of $\mathbf{1 4}$ from 5 suggests that a similar chloro ketone might be produced by ruthenium oxide oxidation of $\mathbf{1}$. The ketone would be transformed to an alcohol analogous to 15 , to which the modified Mosher's method would be applicable. An attempted experiment along this line is in progress.

\section{Experimental}

${ }^{1} \mathrm{H}$ - and ${ }^{13} \mathrm{C}-\mathrm{NMR}$ spectra were measured with JEOL AL-400 and Bruker ARX-400 spectrometers. IR spectra were measured on an FT/IR-420 spectrophotometer. Low resolution (LR)-MS spectra were taken on a JEOL JMSDX 303 spectrometer. GC-MS spectra were taken on a HEWLETT PACKARD 5890 Series II and a JEOL JMS-AM 150. HR-MS spectra were taken under electron impact (EI) conditions or fast atom bombardment (FAB) conditions using a JEOL JMS-SX102A spectrometer.

General Procedure of Ruthenium Tetraoxide Oxidation To a substrate in carbon tetrachloride, acetonitrile, and a phosphate buffer ( $\mathrm{pH} 6.86)$ $(1: 1: 1.5)$ was added sodium periodate $(3.0 \mathrm{eq})$ and ruthenium chloride hydrate $(0.2-0.3 \mathrm{eq})$. The mixture was stirred at $\mathrm{rt}$ (reaction time varied in each reaction), diluted with water, and extracted with dichloromethane $(\times 5)$. The combined organic layer was washed with a $1: 1$ mixture of saturated sodium thiosulfate $\left(\mathrm{Na}_{2} \mathrm{~S}_{2} \mathrm{O}_{3}\right)$ and brine $(1: 1)$, and dried over anhydrous sodium sulfate $\left(\mathrm{Na}_{2} \mathrm{SO}_{4}\right)$, and concentrated.

Preparation of (-)-Carane (4) To a solution of (+)-3-carene (3.1 g, $22.8 \mathrm{mmol})$ in dry THF $(7 \mathrm{ml})$ was added dropwise dimethyl sulfide borane complex $(10.5 \mathrm{ml}, 110.6 \mathrm{mmol}, 4.9 \mathrm{eq})$ at $0{ }^{\circ} \mathrm{C}$ under argon. After the mixture was stirred for $19 \mathrm{~h}$ at $\mathrm{rt}$, water was added to the reaction mixture, and the solution was extracted with dichloromethane $(\times 3)$. The organic layer was washed with brine, dried over anhydrous $\mathrm{Na}_{2} \mathrm{SO}_{4}$, and concentrated to give a residue (yellow oil, $4.5 \mathrm{~g}$ ) which was chromatographed on silica gel to afford (-)-carane (4) $(660.3 \mathrm{mg}, 21 \%) .4:{ }^{1} \mathrm{H}-\mathrm{NMR}\left(400 \mathrm{MHz}, \mathrm{CDCl}_{3}\right) \delta$ : $0.40(1 \mathrm{H}, \mathrm{t}, J=8.0 \mathrm{~Hz}, 6-\mathrm{H}), 0.63(1 \mathrm{H}, \mathrm{m}, 4-\mathrm{H}), 0.67(1 \mathrm{H}, \mathrm{td}, J=13.2,4.4$ $\mathrm{Hz}, 2-\mathrm{H}), 0.71(1 \mathrm{H}, \mathrm{m}, 1-\mathrm{H}), 0.81\left(3 \mathrm{H}, \mathrm{d}, J=6.8 \mathrm{~Hz}, 10-\mathrm{CH}_{3}\right), 0.94(3 \mathrm{H}, \mathrm{s}$, 8- $\left.\mathrm{CH}_{3}\right), 0.97\left(3 \mathrm{H}, \mathrm{s}, 9-\mathrm{CH}_{3}\right), 1.16(1 \mathrm{H}, \mathrm{m}, 3-\mathrm{H}), 1.39(1 \mathrm{H}, \mathrm{m}, 4-\mathrm{H}), 1.73$ $(1 \mathrm{H}, \mathrm{m}, 5-\mathrm{H}), 1.80(1 \mathrm{H}, \mathrm{m}, 5-\mathrm{H}), 1.88(1 \mathrm{H}, \mathrm{ttd}, J=13.2,4.4,2.0 \mathrm{~Hz}, 2-\mathrm{H})$. ${ }^{13} \mathrm{C}-\mathrm{NMR}\left(100 \mathrm{MHz}, \mathrm{CDCl}_{3}\right) \delta: 15.4(\mathrm{C}-8), 17.4(\mathrm{C}-7), 18.1$ (C-6), $19.8(\mathrm{C}-$ 5), 21.0 (C-1), 22.2 (C-10), 28.4 (C-2), 28.7 (C-3), 29.4 (C-9), 31.2 (C-4). GC-MS $m / z 138\left(\mathrm{M}^{+}\right) \cdot[\alpha]_{\mathrm{D}}-41.7^{\circ}(c=2.99$, EtOH $)\left\{\right.$ lit. $[\alpha]_{\mathrm{D}}-47.7^{\circ}$ $\left.(c=3.03, \mathrm{EtOH})^{12)}\right\}$.

Oxidation of (-)-Carane (4) $\quad 4(100.0 \mathrm{mg}, 0.72 \mathrm{mmol})$ was oxidized for $24 \mathrm{~h}$. The reaction afforded a ketone compound $(8)(82.5 \mathrm{mg}, 75 \%) .8:{ }^{1} \mathrm{H}-$ NMR $\left(400 \mathrm{MHz}, \mathrm{CDCl}_{3}\right) \delta: 0.92\left(3 \mathrm{H}, \mathrm{d}, J=6.4 \mathrm{~Hz}, 10-\mathrm{CH}_{3}\right), 1.09(1 \mathrm{H}, \mathrm{m}$, $J=13.6,4.4 \mathrm{~Hz}, 2 \beta-\mathrm{H}), 1.10\left(3 \mathrm{H}, \mathrm{s}, 8-\mathrm{CH}_{3}\right), 1.11\left(3 \mathrm{H}, \mathrm{s}, 9-\mathrm{CH}_{3}\right), 1.36(1 \mathrm{H}$, d, $J=7.6 \mathrm{~Hz}, 6-\mathrm{H}), 1.52(1 \mathrm{H}$, ddd, $J=9.4,8.0,4.4 \mathrm{~Hz}, 1-\mathrm{H}), 1.57(1 \mathrm{H}$, dd, $J=16.8,13.6 \mathrm{~Hz}, 4 \beta-\mathrm{H}), 1.91(1 \mathrm{H}, \mathrm{m}, 3-\mathrm{H}), 2.01(1 \mathrm{H}, \mathrm{tdd}, J=9.6,4.8$, $2.8 \mathrm{~Hz}, 2 \alpha-\mathrm{H}), 2.20(1 \mathrm{H}$, br d, $J=16.8 \mathrm{~Hz}, 4 \alpha-\mathrm{H}) .{ }^{13} \mathrm{C}-\mathrm{NMR}(100 \mathrm{MHz}$, $\left.\mathrm{CDCl}_{3}\right) \delta: 16.6(\mathrm{C}-8), 21.1(\mathrm{C}-10), 27.5$ (C-2), 27.7 (C-7), 29.1 (C-9), 30.5 (C-1), 34.1 (C-3), 34.5 (C-6), 48.3 (C-4), 209.8 (C-5).

Reduction of 8 To a solution of $8(39.6 \mathrm{mg}, 0.24 \mathrm{mmol})$ in THF/ dimethoxyethane $(1: 1,5.2 \mathrm{ml})$ was added diisobutylaluminum hydride $(0.35 \mathrm{ml}$ of a $1.0 \mathrm{M}$ solution in toluene, $0.35 \mathrm{mmol})$ at $-60^{\circ} \mathrm{C}$. After stirring for $5 \mathrm{~min}$ at $-60^{\circ} \mathrm{C}$, the reaction was quenched with methanol $(18 \mu \mathrm{l})$, and the mixture was diluted with ether. The ethereal solution was washed with saturated potassium sodium tartrate solution, and dried over $\mathrm{Na}_{2} \mathrm{SO}_{4}$. Filtration and concentration afforded the alcohol $(9)$ as white crystals $(42.7 \mathrm{mg})$. 9: ${ }^{1} \mathrm{H}-\mathrm{NMR}\left(400 \mathrm{MHz}, \mathrm{CDCl}_{3}\right) \delta: 0.87\left(3 \mathrm{H}, \mathrm{d}, J=6.4 \mathrm{~Hz}, 10-\mathrm{CH}_{3}\right), 1.04$ $(3 \mathrm{H}, \mathrm{s}), 1.15(3 \mathrm{H}, \mathrm{s}), 4.29(1 \mathrm{H}, \mathrm{dt}, J=10.8,7.2 \mathrm{~Hz}, 5-\mathrm{H})$.

Condensation of $(\boldsymbol{R})$-MTPA with $9 \quad \mathbf{9}(15.0 \mathrm{mg}), 37.6 \mathrm{mg}$ of 2,4,6-trinitrochlorobenzene and $24.7 \mathrm{mg}$ of $(R)$-MTPA were dissolved in $1.5 \mathrm{ml}$ of pyridine. After the mixture was stirred for $10.5 \mathrm{~h}$, ether was added, and organic layer was washed with $0.1 \mathrm{M} \mathrm{NaOH}$, water, saturated aqueous copper(II) sulfate and brine, dried over $\mathrm{Na}_{2} \mathrm{SO}_{4}$ and concentrated to yield a residue (yellow oil, $30.3 \mathrm{mg}$ ). The crude product was chromatographed on silica gel to afford the ester (10) $(19.9 \mathrm{mg}, 64.2 \%$ \{two steps $\})$ as a colorless oil. 10: ${ }^{1} \mathrm{H}-\mathrm{NMR}\left(400 \mathrm{MHz}, \mathrm{CDCl}_{3}\right) \delta: 0.83(1 \mathrm{H}, \mathrm{m}, 2 \beta-\mathrm{H}), 0.89(3 \mathrm{H}, \mathrm{d}$, $\left.J=6.8 \mathrm{~Hz}, 10-\mathrm{CH}_{3}\right), 0.92(1 \mathrm{H}, \mathrm{m}, 4 \beta-\mathrm{H}), 1.00(1 \mathrm{H}, \mathrm{m}, 6-\mathrm{H}), 1.02(3 \mathrm{H}, \mathrm{s}, 9-$ $\left.\mathrm{CH}_{3}\right), 1.03\left(3 \mathrm{H}, \mathrm{s}, 8-\mathrm{CH}_{3}\right), 1.11(1 \mathrm{H}, \mathrm{td}, J=9.2,4.0 \mathrm{~Hz}, 1-\mathrm{H}), 1.42(1 \mathrm{H}, \mathrm{m}$, $3-\mathrm{H}), 1.81(1 \mathrm{H}, \mathrm{ddt}, J=12.6,7.8,2.3 \mathrm{~Hz}, 4 \alpha-\mathrm{H}), 1.90(1 \mathrm{H}$, dddd, $J=14.5$, 9.7, $4.8,1.9 \mathrm{~Hz}, 2 \alpha-\mathrm{H}), 3.56\left(3 \mathrm{H}, \mathrm{q}, J=0.8 \mathrm{~Hz},-\mathrm{OCH}_{3}\right), 5.71(1 \mathrm{H}, \mathrm{dt}$, $J=10.8,7.6 \mathrm{~Hz}, 5-\mathrm{H}), 7.39-7.58(5 \mathrm{H}, \mathrm{Ph}-\mathrm{H}) .{ }^{13} \mathrm{C}-\mathrm{NMR}\left(100 \mathrm{MHz}, \mathrm{CDCl}_{3}\right)$ $\delta: 16.3(\mathrm{C}-8), 21.0(\mathrm{C}-7), 21.3(\mathrm{C}-10), 22.6(\mathrm{C}-6), 26.0(\mathrm{C}-1), 27.6(\mathrm{C}-2)$, 29.7 (C-9), $30.4(\mathrm{C}-3), 36.1(\mathrm{C}-4), 55.2\left(-\mathrm{OCH}_{3}\right), 75.9(\mathrm{C}-5), 127.6,128.3$, 129.4, $132.8(\mathrm{Ph}), 166.3(\mathrm{C}=\mathrm{O})$. HR-MS (EI) $\mathrm{m} / \mathrm{z}: 370.1764$ (Calcd for $\left.\mathrm{C}_{20} \mathrm{H}_{25} \mathrm{~F}_{3} \mathrm{O}_{3}: 370.1756\right)$.

Condensation of (S)-MTPA with 9 Condensation of compound 9 (18.1 mg, containing solvents) with $(S)$-MTPA in the same manner afforded the desired product (11) $(19.0 \mathrm{mg}, 50 \%$ two steps $\})$ as a colorless oil. 11: ${ }^{1} \mathrm{H}-\mathrm{NMR}\left(400 \mathrm{MHz}, \mathrm{CDCl}_{3}\right) \delta: 0.81(1 \mathrm{H}, \mathrm{m}, J=15.6 \mathrm{~Hz}, 2 \beta-\mathrm{H}), 0.84(3 \mathrm{H}, \mathrm{s}$, $\left.8-\mathrm{CH}_{3}\right), 0.90\left(3 \mathrm{H}, \mathrm{d}, J=6.8 \mathrm{~Hz}, 10-\mathrm{CH}_{3}\right), 0.93\left(3 \mathrm{H}, \mathrm{s}, 9-\mathrm{CH}_{3}\right), 0.98(1 \mathrm{H}, \mathrm{m}$, $J=7.2 \mathrm{~Hz}, 4 \beta-\mathrm{H}), 1.02(1 \mathrm{H}, \mathrm{m}, 6-\mathrm{H}), 1.06(1 \mathrm{H}, \mathrm{m}, 1-\mathrm{H}), 1.42(1 \mathrm{H}, \mathrm{m}, 3-\mathrm{H})$, $1.83(1 \mathrm{H}, \mathrm{dt}, J=8.0,2.4 \mathrm{~Hz}, 4 \alpha-\mathrm{H}), 1.88(1 \mathrm{H}, \mathrm{dddd}, J=14.2,9.4,5.0$, $1.8 \mathrm{~Hz}, 2 \alpha-\mathrm{H}), 3.57\left(3 \mathrm{H}, \mathrm{q}, J=0.8 \mathrm{~Hz},-\mathrm{OCH}_{3}\right), 5.65(1 \mathrm{H}, \mathrm{dt}, J=10.8$, $7.6 \mathrm{~Hz}, 5-\mathrm{H}), 7.39-7.57(5 \mathrm{H}, \mathrm{Ph}-\mathrm{H}) .{ }^{13} \mathrm{C}-\mathrm{NMR}\left(100 \mathrm{MHz}, \mathrm{CDCl}_{3}\right) \delta: 16.1$ (C-8), 21.0 (C-7), 21.3 (C-10), 22.4 (C-6), 26.1 (C-1), 27.6 (C-2), 29.5 (C9), 30.4 (C-3), $36.4(\mathrm{C}-4), 55.4\left(-\mathrm{OCH}_{3}\right), 76.1(\mathrm{C}-5), 127.4,128.3,129.4$, $132.8(\mathrm{Ph}), 166.2(\mathrm{C}=\mathrm{O})$. HR-MS (EI) $\mathrm{m} / \mathrm{z}: 370.1751$ (Calcd for $\left.\mathrm{C}_{20} \mathrm{H}_{25} \mathrm{~F}_{3} \mathrm{O}_{3}: 370.1756\right)$.

Oxidation of Cyclosativene Cyclosativene (5) $(100 \mathrm{mg}, 0.49 \mathrm{mmol})$ was oxidized for $24 \mathrm{~h}$. After the crude product was purified by preparative TLC, 13 (16.2 mg, $0.074 \mathrm{mmol}, 15 \%), \mathbf{1 4}(8.3 \mathrm{mg}, 0.034 \mathrm{mmol}, 7 \%)$ and $\mathbf{1 6}$ $(6.5 \mathrm{mg}, 0.025 \mathrm{mmol}, 5 \%)$ were obtained as colorless oils. 13: ${ }^{1} \mathrm{H}-\mathrm{NMR}$ $\left(400 \mathrm{MHz}, \mathrm{CDCl}_{3}\right) \delta: 0.73(1 \mathrm{H}, \mathrm{brd}, J=5.6 \mathrm{~Hz}, \mathrm{H}-7), 0.77\left(3 \mathrm{H}, \mathrm{s}, 11-\mathrm{CH}_{3}\right)$, $0.87(1 \mathrm{H}, \mathrm{m}, 9-\mathrm{H}), 0.89\left(3 \mathrm{H}, \mathrm{d}, J=7.0 \mathrm{~Hz}, 13-\mathrm{CH}_{3}\right), 0.92(3 \mathrm{H}, \mathrm{d}, J=7.0 \mathrm{~Hz}$, $\left.14-\mathrm{CH}_{3}\right), 1.00\left(3 \mathrm{H}, \mathrm{s}, 15-\mathrm{CH}_{3}\right), 1.03(1 \mathrm{H}$, brd, $J=11.6 \mathrm{~Hz}, 10 \alpha-\mathrm{H}), 1.40$ $(1 \mathrm{H}, \mathrm{m}, 3 \alpha-\mathrm{H}), 1.44(1 \mathrm{H}, \mathrm{m}, 4 \beta-\mathrm{H}), 1.52(1 \mathrm{H}, \mathrm{m}, 3 \beta-\mathrm{H}), 1.52(1 \mathrm{H}, \mathrm{m}, 4 \alpha-$ $\mathrm{H}), 1.53(1 \mathrm{H}, \mathrm{brs}, 1-\mathrm{H}), 1.68(1 \mathrm{H}, \mathrm{brd}, J=11.6 \mathrm{~Hz}, 10 \beta-\mathrm{H}), 1.70(1 \mathrm{H}, \mathrm{m}$, $J=7.0 \mathrm{~Hz}, 12-\mathrm{H}), 1.77(1 \mathrm{H}, \mathrm{br} \mathrm{s}, 6-\mathrm{H}) .{ }^{13} \mathrm{C}-\mathrm{NMR}\left(100 \mathrm{MHz}, \mathrm{CDCl}_{3}\right) \delta: 10.4$ (C-15), 16.0 (C-13), 16.4 (C-14), 19.0 (C-11), 20.0 (C-9), 22.0 (C-8), 22.3 (C-7), 29.0 (C-3), 30.9 (C-4), 31.0 (C-10), 35.6 (C-12), 41.3 (C-1), 43.8 (C- 
2), 49.3 (C-6), 74.5 (C-5). HR-MS (EI) $m / z$ : 220.1835 (Calcd for $\mathrm{C}_{15} \mathrm{H}_{24} \mathrm{O}$ : 220.1827). 14: ${ }^{1} \mathrm{H}-\mathrm{NMR}\left(400 \mathrm{MHz}, \mathrm{CDCl}_{3}\right) \delta: 0.70(1 \mathrm{H}, \mathrm{qd}, J=13.6$, $3.6 \mathrm{~Hz}, 4 \beta-\mathrm{H}), 0.91\left(3 \mathrm{H}, \mathrm{d}, J=6.6 \mathrm{~Hz}, 13-\mathrm{CH}_{3}\right), 1.00\left(3 \mathrm{H}, \mathrm{s}, 11-\mathrm{CH}_{3}\right), 1.03$ $\left(3 \mathrm{H}, \mathrm{d}, J=6.6 \mathrm{~Hz}, 14-\mathrm{CH}_{3}\right), 1.15(1 \mathrm{H}, \mathrm{td}, J=13.6,4.4 \mathrm{~Hz}, 3 \alpha-\mathrm{H}), 1.19(1 \mathrm{H}$, $\mathrm{m}, 5-\mathrm{H}), 1.47(1 \mathrm{H}$, septd, $J=6.6,2.2 \mathrm{~Hz}, 12-\mathrm{H}), 1.60(1 \mathrm{H}, \mathrm{br} \mathrm{d}, J=13.6 \mathrm{~Hz}$, $4 \alpha-\mathrm{H}), 1.88(1 \mathrm{H}, \mathrm{dt}, J=13.6,3.6 \mathrm{~Hz}, 3 \beta-\mathrm{H}), 2.05(1 \mathrm{H}, \mathrm{dd}, J=11.0,3.0 \mathrm{~Hz}$, $10 \mathrm{~b}-\mathrm{H}), 2.14(1 \mathrm{H}, \mathrm{d}, J=11.0 \mathrm{~Hz}, 10 \alpha-\mathrm{H}), 2.46(1 \mathrm{H}$, br d, $J=3.0 \mathrm{~Hz}, 1-\mathrm{H})$, $2.76\left(1 \mathrm{H}\right.$, br s, 6-H), $2.76(1 \mathrm{H}$, brs, $9-\mathrm{H}), 3.80(1 \mathrm{H}, \mathrm{brs}, 7-\mathrm{H}) .{ }^{13} \mathrm{C}-\mathrm{NMR}$ $\left(100 \mathrm{MHz}, \mathrm{CDCl}_{3}\right) \delta: 20.7$ (C-13), $21.1(\mathrm{C}-14), 22.1$ (C-11), 22.7 (C-4), 30.7 (C-12), 32.6 (C-10), 33.9 (C-3), 44.1 (C-5), 46.9 (C-2), 48.1 (C-1), 50.2 (C-6), 56.7 (C-7), 61.2 (C-9), 219.5 (C-8). ${ }^{1} \mathrm{H}-\mathrm{NMR}\left(400 \mathrm{MHz}, \mathrm{C}_{6} \mathrm{D}_{6}\right) \delta$ : $0.47(1 \mathrm{H}, \mathrm{qd}, J=13.4,3.4 \mathrm{~Hz}, 4 \beta-\mathrm{H}), 0.75(1 \mathrm{H}, \mathrm{td}, J=13.4,3.4 \mathrm{~Hz}, 3 \alpha-\mathrm{H})$, $0.76\left(3 \mathrm{H}, \mathrm{d}, J=6.4 \mathrm{~Hz}, 13-\mathrm{CH}_{3}\right), 0.77(1 \mathrm{H}, \mathrm{m}, 5-\mathrm{H}), 0.77\left(3 \mathrm{H}, \mathrm{s}, 11-\mathrm{CH}_{3}\right)$, $1.00\left(3 \mathrm{H}, \mathrm{d}, J=6.4 \mathrm{~Hz}, 14-\mathrm{CH}_{3}\right), 1.22(1 \mathrm{H}, \mathrm{m}, 12-\mathrm{H}), 1.23(1 \mathrm{H}, \mathrm{dd}, J=13.4$, $3.4 \mathrm{~Hz}, 4 \alpha-\mathrm{H}), 1.54(1 \mathrm{H}, \mathrm{q}, J=1.6 \mathrm{~Hz}, 1-\mathrm{H}), 1.61(1 \mathrm{H}, \mathrm{dd}, J=11.0,1.6 \mathrm{~Hz}$, $10 \beta-\mathrm{H}), 1.83(1 \mathrm{H}, \mathrm{dt}, J=13.4,3.4 \mathrm{~Hz}, 3 \beta-\mathrm{H}), 1.89(1 \mathrm{H}, \mathrm{d}, J=11.0 \mathrm{~Hz}, 10 \alpha-$ $\mathrm{H}), 2.56(1 \mathrm{H}$, br d, $J=3.2 \mathrm{~Hz}, 6-\mathrm{H}), 2.77(1 \mathrm{H}, \mathrm{br} \mathrm{s}, 9-\mathrm{H}), 3.68(1 \mathrm{H}, \mathrm{br} \mathrm{s}, 7-\mathrm{H})$. ${ }^{13} \mathrm{C}-\mathrm{NMR}\left(100 \mathrm{MHz}, \mathrm{C}_{6} \mathrm{D}_{6}\right) \delta: 20.8(\mathrm{C}-13), 21.3(\mathrm{C}-14), 22.2$ (C-11), 22.7 (C-4), 30.8 (C-12), 32.7 (C-10), 34.0 (C-3), 44.2 (C-5), 46.6 (C-2), 48.1 (C1), 50.5 (C-6), 57.1 (C-7), 61.4 (C-9), 216.8 (C-8). GC-MS m/z (rel. int. \%): $242\left(\mathrm{M}^{+}+2,33\right), 240$ (100). HR-MS (EI) $\mathrm{m} / \mathrm{z}: 240.1284$ (Calcd for $\mathrm{C}_{14} \mathrm{H}_{21} \mathrm{ClO}: 240.1281$ ). IR (liquid film) $1748 \mathrm{~cm}^{-1}$. 16: ${ }^{1} \mathrm{H}-\mathrm{NMR}(400 \mathrm{MHz}$, $\left.\mathrm{CDCl}_{3}\right) \delta: 0.88\left(3 \mathrm{H}, \mathrm{d}, J=6.6 \mathrm{~Hz}, 13-\mathrm{CH}_{3}\right), 1.03\left(3 \mathrm{H}, \mathrm{d}, J=6.6 \mathrm{~Hz}, 14-\mathrm{CH}_{3}\right)$, $1.17\left(3 \mathrm{H}, \mathrm{s}, 11-\mathrm{CH}_{3}\right), 1.17(1 \mathrm{H}, \mathrm{m}, 4 \beta-\mathrm{H}), 1.28(1 \mathrm{H}, \mathrm{m}, 5-\mathrm{H}), 1.47(1 \mathrm{H}, \mathrm{m}$, $12-\mathrm{H}), 1.51(1 \mathrm{H}, \mathrm{m}, J=14.6 \mathrm{~Hz}, 3 \alpha-\mathrm{H}), 1.59\left(3 \mathrm{H}, \mathrm{s}, 15-\mathrm{CH}_{3}\right), 1.89(1 \mathrm{H}, \mathrm{dd}$, $J=17.6,4.0 \mathrm{~Hz}, 4 \alpha-\mathrm{H}), 1.96(1 \mathrm{H}, \mathrm{dd}, J=8.0,2.6 \mathrm{~Hz}, 1-\mathrm{H}), 2.11(1 \mathrm{H}, \mathrm{ddd}$, $J=14.6,4.0,2.0 \mathrm{~Hz}, 3 \beta-\mathrm{H}), 2.55(1 \mathrm{H}, \mathrm{d}, J=19.8 \mathrm{~Hz}, 10 \beta-\mathrm{H}), 2.93(1 \mathrm{H}, \mathrm{br} \mathrm{s}$, 6-H), $3.11(1 \mathrm{H}, \mathrm{dd}, J=19.8,8.0 \mathrm{~Hz}, 10 \alpha-\mathrm{H}) .{ }^{13} \mathrm{C}-\mathrm{NMR}\left(100 \mathrm{MHz}, \mathrm{CDCl}_{3}\right)$ $\delta$ : 20.5 (C-13), 21.1 (C-14), 21.3 (C-15), 23.2 (C-11), 24.5 (C-4), 29.4 (C12), 34.2 (C-2), 34.4 (C-10), 34.9 (C-3), 39.9 (C-1), 47.7 (C-6), 48.5 (C-5), 110.1 (C-8), 167.1 (C-9), 168.0 (C-7). HR-MS (FAB): $m / z: 267.1597$ (Calcd for $\mathrm{C}_{15} \mathrm{H}_{22} \mathrm{O}_{4}: 267.1596\left[\mathrm{M}^{+}+\mathrm{H}\right]$ ). IR (liquid film) $1749 \mathrm{~cm}^{-1}$.

Reduction of 14 Sodium borohydride $(5.8 \mathrm{mg})$ was added to $0.8 \mathrm{mg}$ of 14 in $0.1 \mathrm{ml}$ of $\mathrm{MeOH}$. After the mixture was stirred for $30 \mathrm{~min}$ at $\mathrm{rt}$, the reaction mixture was concentrated by applying nitrogen. The residue was diluted with water, and the mixture was extracted with dichloromethane $(\times 3)$. The combined organic layer was washed with brine, dried over anhydrous $\mathrm{Na}_{2} \mathrm{SO}_{4}$, and concentrated to yield $1.1 \mathrm{mg}$ (containing solvents) of $15:{ }^{1} \mathrm{H}-$ NMR $\left(400 \mathrm{MHz}, \mathrm{CDCl}_{3}\right) \delta: 0.91\left(3 \mathrm{H}, \mathrm{d}, J=7.0 \mathrm{~Hz}, 13-\mathrm{CH}_{3}\right), 0.94(3 \mathrm{H}, \mathrm{s}$, $\left.11-\mathrm{CH}_{3}\right), 1.03\left(3 \mathrm{H}, \mathrm{d}, J=7.0 \mathrm{~Hz}, 14-\mathrm{CH}_{3}\right), 1.07(1 \mathrm{H}, \mathrm{m}, 5-\mathrm{H}), 1.09(1 \mathrm{H}, \mathrm{td}$, $J=13.4,4.0 \mathrm{~Hz}, 3 \alpha-\mathrm{H}), 1.32(1 \mathrm{H}, \mathrm{qd}, J=13.4,4.0 \mathrm{~Hz}, 4 \beta-\mathrm{H}), 1.55(1 \mathrm{H}, \mathrm{brd}$, $J=13.4 \mathrm{~Hz}, 4 \alpha-\mathrm{H}), 1.56(1 \mathrm{H}, \mathrm{m}, 12-\mathrm{H}), 1.59(1 \mathrm{H}, \mathrm{d}, J=10.8 \mathrm{~Hz}, 10 \beta-\mathrm{H})$, $1.63(1 \mathrm{H}$, br s, $1-\mathrm{H}), 1.65(1 \mathrm{H}$, brd, $J=13.4 \mathrm{~Hz}, 3 \beta-\mathrm{H}), 1.72(1 \mathrm{H}, \mathrm{d}$, $J=10.8 \mathrm{~Hz}, 10 \alpha-\mathrm{H}), 2.47(1 \mathrm{H}, \mathrm{m}, 9-\mathrm{H}), 2.48(1 \mathrm{H}, \mathrm{m}, 6-\mathrm{H}), 3.81(1 \mathrm{H}, \mathrm{d}$, $J=3.6 \mathrm{~Hz}, 8-\mathrm{H}), 4.25(1 \mathrm{H}, \mathrm{d}, J=4.2 \mathrm{~Hz}, 7-\mathrm{H}) .{ }^{13} \mathrm{C}-\mathrm{NMR}\left(100 \mathrm{MHz}, \mathrm{CDCl}_{3}\right)$ $\delta$ : 20.7 (C-13), 21.3 (C-14), 23.8 (C-4), 30.0 (C-11), 30.9 (C-10), 30.9 (C12), 33.0 (C-3), 36.1 (C-2), 43.8 (C-5), 50.6 (C-1), 51.1 (C-6), 54.3 (C-9), 56.5 (C-7), 80.6 (C-8). HR-MS (EI) $m / z: 242.1439$ (Calcd for $\mathrm{C}_{14} \mathrm{H}_{23} \mathrm{ClO}$ : 242.1437).

Preparation of Laurinterol Methyl Ether (6) A solution of laurinterol $(300.0 \mathrm{mg}, 1.0 \mathrm{mmol})$ in dry THF $(10 \mathrm{ml})$ was treated with sodium hydride ( $60 \%$ dispersion in mineral oil; $79.9 \mathrm{mg}, 2.0 \mathrm{mmol}$ ) under nitrogen. The mixture was stirred at $\mathrm{rt}$ for $30 \mathrm{~min}$. Then, iodomethane $(0.31 \mathrm{ml}, 5.0 \mathrm{mmol})$ was added to the mixture. After the mixture was stirred for $10 \mathrm{~h}$ at rt, water and ether were added and the organic layer was washed with $1 \mathrm{~m} \mathrm{NaOH}$, water and brine. The organic layer was dried over anhydrous $\mathrm{Na}_{2} \mathrm{SO}_{4}$ and concentrated to give a residue $(292.5 \mathrm{mg})$ which was chromatographed on silica gel to afford $6(229.1 \mathrm{mg}, 74 \%)$ as white crystals. 6: ${ }^{1} \mathrm{H}-\mathrm{NMR}(400 \mathrm{MHz}$, $\left.\mathrm{CDCl}_{3}\right) \delta: 0.47-0.52(2 \mathrm{H}, \mathrm{m}$, overlap), $1.06(1 \mathrm{H}, \mathrm{dt}, J=7.6,4.0 \mathrm{~Hz}), 1.17$ $(1 \mathrm{H}, \mathrm{td}, J=12.2,8.2 \mathrm{~Hz}), 1.29(3 \mathrm{H}, \mathrm{s}), 1.35(3 \mathrm{H}, \mathrm{s}), 1.60(1 \mathrm{H}, \mathrm{dd}, J=12.4$, $8.0 \mathrm{~Hz}), 1.91(1 \mathrm{H}, \mathrm{m}), 2.16(1 \mathrm{H}, \mathrm{dd}, J=13.6,8.0 \mathrm{~Hz}), 2.35(3 \mathrm{H}, \mathrm{s}$, benzylic$\left.\mathrm{CH}_{3}\right), 3.76\left(3 \mathrm{H}, \mathrm{s},-\mathrm{OCH}_{3}\right), 6.72(1 \mathrm{H}, \mathrm{s}, \mathrm{Ph}-\mathrm{H}), 7.67(1 \mathrm{H}, \mathrm{s}, \mathrm{Ph}-\mathrm{H})$.

Oxidation of Laurinterol Methyl Ether (6) Laurinterol methyl ether (6) $(19.6 \mathrm{mg}, 0.063 \mathrm{mmol})$ was oxidized for $26 \mathrm{~h}$. After the crude product was purified by preparative TLC, $18(6.5 \mathrm{mg}, 0.020 \mathrm{mmol}, 32 \%)$ was obtained. 18: ${ }^{1} \mathrm{H}-\mathrm{NMR}\left(400 \mathrm{MHz}, \mathrm{CDCl}_{3}\right) \delta: 1.59\left(3 \mathrm{H}, \mathrm{s}, \mathrm{CH}_{3}\right), 1.65(3 \mathrm{H}, \mathrm{d}$, $J=0.8 \mathrm{~Hz}$, allylic- $\left.\mathrm{CH}_{3}\right), 1.69(1 \mathrm{H}, \mathrm{dt}, J=13.6,4.8 \mathrm{~Hz}), 2.35(1 \mathrm{H}, \mathrm{dt}, J=16.8$, $4.8 \mathrm{~Hz}), 2.37\left(3 \mathrm{H}, \mathrm{s}\right.$, benzylic- $\left.\mathrm{CH}_{3}\right), 2.53(1 \mathrm{H}, \mathrm{ddd}, J=17.4,12.8,5.2 \mathrm{~Hz})$, $2.71(1 \mathrm{H}, \mathrm{td}, J=12.8,4.8 \mathrm{~Hz}), 3.72\left(3 \mathrm{H}, \mathrm{s},-\mathrm{OCH}_{3}\right), 5.88(1 \mathrm{H}, \mathrm{s}$, olefin- $\mathrm{H})$, $6.72(1 \mathrm{H}, \mathrm{s}, \mathrm{Ph}-\mathrm{H}), 7.34$ (1H, s, Ph-H). ${ }^{13} \mathrm{C}-\mathrm{NMR}\left(100 \mathrm{MHz}, \mathrm{CDCl}_{3}\right) \delta: 20.9$ (q), $22.7(\mathrm{q}), 23.3(\mathrm{q}), 34.6(\mathrm{t}), 35.0(\mathrm{t}), 42.5(\mathrm{~s}), 55.0(\mathrm{q}), 113.7(\mathrm{~d}), 115.4$ (s), 125.8 (d), 131.1 (d), 133.1 (s), 137.6 (s), 156.3 (s), 169.4 (s), 198.9 (s). MS $m / z$ (rel. int. \%): $324\left(\mathrm{M}^{+}+2,99.8\right), 322$ (100). HR-MS (EI) $\mathrm{m} / \mathrm{z}$ : 322.0546 (Calcd for $\mathrm{C}_{16} \mathrm{H}_{19} \mathrm{BrO}_{2}: 322.0568$ ). IR (liquid film) $1660 \mathrm{~cm}^{-1}$.
Preparation of Thujopsane (7) To a solution of thujopsene (2.4 g, 11.7 $\mathrm{mmol})$ in dry THF $(7 \mathrm{ml})$ was added dimethyl sulfide borane complex $(2.3$ $\mathrm{ml}, 24.2 \mathrm{mmol}$ ) at $0{ }^{\circ} \mathrm{C}$ under argon. After the mixture was stirred for $15 \mathrm{~h}$ at $\mathrm{rt}$, water was added to the reaction mixture, and the solution was extracted with dichloromethane $(\times 3)$. The organic layer was washed with brine, dried over anhydrous $\mathrm{Na}_{2} \mathrm{SO}_{4}$ and concentrated to give a residue (yellow oil, 1.7 $\mathrm{g})$, which was chromatographed on silica gel to afford $7(134.7 \mathrm{mg}, 6 \%)$ as a colorless oil. 7: ${ }^{1} \mathrm{H}-\mathrm{NMR}\left(400 \mathrm{MHz}, \mathrm{CDCl}_{3}\right) \delta: 0.012(1 \mathrm{H}, \mathrm{t}, J=5.2 \mathrm{~Hz}, 1-$ $\mathrm{H}), 0.39(1 \mathrm{H}, \mathrm{dd}, J=10.0,5.2 \mathrm{~Hz}, 1-\mathrm{H}) 0.51\left(3 \mathrm{H}, \mathrm{s}, 8-\mathrm{CH}_{3}\right), 0.80(1 \mathrm{H}, \mathrm{dd}$, $J=10.0,5.6 \mathrm{~Hz}, 1 \mathrm{a}-\mathrm{H}), 0.88(1 \mathrm{H}, \mathrm{m}, 4 \beta-\mathrm{H}), 1.0\left(3 \mathrm{H}, \mathrm{s}, 8-\mathrm{CH}_{3}\right), 1.0(3 \mathrm{H}, \mathrm{d}$, $\left.J=6.7 \mathrm{~Hz}, 2-\mathrm{CH}_{3}\right), 1.0(1 \mathrm{H}, \mathrm{t}, J=12.8 \mathrm{~Hz}, 3 \beta-\mathrm{H}), 1.0(1 \mathrm{H}, \mathrm{m}, 4 \alpha-\mathrm{H}), 1.1$ $\left(3 \mathrm{H}, \mathrm{s}, 4 \mathrm{a}-\mathrm{CH}_{3}\right), 1.1(1 \mathrm{H}, \mathrm{d}, J=14.2 \mathrm{~Hz}, 5 \alpha-\mathrm{H}), 1.2(1 \mathrm{H}, \mathrm{dd}, J=12.8,6.0 \mathrm{~Hz}$, $3 \alpha-\mathrm{H}), 1.2(1 \mathrm{H}, \mathrm{t}, J=14.2 \mathrm{~Hz}, 7 \beta-\mathrm{H}), 1.4(1 \mathrm{H}, \mathrm{d}, J=14.2 \mathrm{~Hz}, 7 \alpha-\mathrm{H}), 1.5$ $(1 \mathrm{H}, \mathrm{d}, J=14.2 \mathrm{~Hz}, 6 \beta-\mathrm{H}), 1.5(1 \mathrm{H}, \mathrm{m}, 2-\mathrm{H}), 1.7(1 \mathrm{H}, \mathrm{td}, J=14.2,3.2 \mathrm{~Hz}$, $5 \beta-\mathrm{H}), 1.8(1 \mathrm{H}, \mathrm{tt}, J=14.2,3.2 \mathrm{~Hz}, 6 \alpha-\mathrm{H}) .{ }^{13} \mathrm{C}-\mathrm{NMR}\left(100 \mathrm{MHz}, \mathrm{CDCl}_{3}\right) \delta$ : 13.1 (C-1), $19.0(\mathrm{C}-6), 24.4\left(2-\mathrm{CH}_{3}\right), 25.2(\mathrm{C}-1 \mathrm{a}), 27.1\left(8-\mathrm{CH}_{3}\right), 27.5(\mathrm{C}-3)$, $29.3\left(8-\mathrm{CH}_{3}\right), 29.6\left(4 \mathrm{a}-\mathrm{CH}_{3}\right), 31.1(\mathrm{C}-2), 31.6(\mathrm{C}-4 \mathrm{a}), 32.4(\mathrm{C}-8 \mathrm{a}), 33.4(\mathrm{C}-$ 8), 36.6 (C-5), 38.6 (C-4), 40.8 (C-7). GC-MS $m / z 206\left(\mathrm{M}^{+}\right)$.

Oxidation of Thujopsane (7) $7(25.2 \mathrm{mg}, 0.12 \mathrm{mmol})$ was oxidized for $24 \mathrm{~h}$. The ${ }^{1} \mathrm{H}$-NMR spectrum of the crude product $(32.1 \mathrm{mg})$ indicated that ca. $30 \%$ yield 19 was produced. The crude product was methylated with diazomethane in an ether solution. After the solvent was removed, the residue was chromatographed on silica gel to afford 20 (5.9 mg, 18\% from thujopsane). In another experiment, a small amount of 19 was isolated from an oxidation product by chromatography on silica gel. 19: ${ }^{1} \mathrm{H}-\mathrm{NMR}(400 \mathrm{MHz}$, $\left.\mathrm{CDCl}_{3}\right) \delta: 0.69\left(3 \mathrm{H}, \mathrm{s}, 8-\mathrm{CH}_{3}\right), 0.99\left(3 \mathrm{H}, \mathrm{s}, 4 \mathrm{a}-\mathrm{CH}_{3}\right), 1.16(1 \mathrm{H}, \mathrm{dd}, J=5.9$, $4.8 \mathrm{~Hz}, 1-\mathrm{H}), 1.24\left(3 \mathrm{H}, \mathrm{s}, 8-\mathrm{CH}_{3}\right), 1.26(1 \mathrm{H}, \mathrm{t}, J=13.6 \mathrm{~Hz}, 5 \mathrm{~b}-\mathrm{H}), 1.32(1 \mathrm{H}$, td, $J=13.6,3.6 \mathrm{~Hz}, 7 \alpha-\mathrm{H}), 1.45(1 \mathrm{H}, \mathrm{dt}, J=13.6,3.6 \mathrm{~Hz}, 6 \alpha-\mathrm{H}), 1.52(1 \mathrm{H}$, dq, $J=13.6,3.0 \mathrm{~Hz}, 7 \beta-\mathrm{H}), 1.70(1 \mathrm{H}$, br s, $1-\mathrm{H}), 1.74(1 \mathrm{H}, \mathrm{q}, J=13.6 \mathrm{~Hz}$, $6 \beta-\mathrm{H}), 1.74(1 \mathrm{H}, \mathrm{m}, 1 \mathrm{a}-\mathrm{H}), 1.94(1 \mathrm{H}, \mathrm{dq}, J=13.6,2.8 \mathrm{~Hz}, 5 \beta-\mathrm{H}), 2.04(1 \mathrm{H}$, d, $J=12.8 \mathrm{~Hz}, 4-\mathrm{H}), 2.35\left(3 \mathrm{H}, \mathrm{s}, 2-\mathrm{CH}_{3}\right), 3.05(1 \mathrm{H}, \mathrm{d}, J=12.8 \mathrm{~Hz}, 4-\mathrm{H}) .{ }^{13} \mathrm{C}-$ NMR $\left(100 \mathrm{MHz}, \mathrm{CDCl}_{3}\right) \delta: 17.0(\mathrm{C}-1), 18.4(\mathrm{C}-6), 26.8\left(4 \mathrm{a}-\mathrm{CH}_{3}\right), 29.0(8-$ $\left.\mathrm{CH}_{3}\right), 29.6\left(8-\mathrm{CH}_{3}\right), 32.4\left(2-\mathrm{CH}_{3}\right), 32.6(\mathrm{C}-1 \mathrm{a}), 36.0(\mathrm{C}-8), 38.0(\mathrm{C}-4 \mathrm{a}), 39.9$ (C-5), 40.7 (C-7), 43.5 (C-4), 47.9 (C-8a), 177.0 (C-3), 207.8 (C-2). HR-MS (EI) $m / z: 252.1706\left(\right.$ Calcd for $\left.\mathrm{C}_{15} \mathrm{H}_{24} \mathrm{O}_{3}: 252.1725\right)$. 20: ${ }^{1} \mathrm{H}-\mathrm{NMR}(400 \mathrm{MHz}$, $\left.\mathrm{CDCl}_{3}\right) \delta: 0.68\left(3 \mathrm{H}, \mathrm{s}, 8-\mathrm{CH}_{3}\right), 0.92\left(3 \mathrm{H}, \mathrm{s}, 4 \mathrm{a}-\mathrm{CH}_{3}\right), 1.14(1 \mathrm{H}, \mathrm{dd}, J=6.8$, $4.4 \mathrm{~Hz}, 1-\mathrm{H}), 1.24\left(3 \mathrm{H}, \mathrm{s}, 8-\mathrm{CH}_{3}\right), 1.25(1 \mathrm{H}, \mathrm{m}, 5-\mathrm{H}), 1.31(1 \mathrm{H}, \mathrm{td}, J=13.2$, $4.0 \mathrm{~Hz}, 7-\mathrm{H}), 1.44(1 \mathrm{H}, \mathrm{dt}, J=14.0,3.6 \mathrm{~Hz}, 6-\mathrm{H}), 1.51(1 \mathrm{H}, \mathrm{dbrd}, J=13.0$, $2.8 \mathrm{~Hz}, 7-\mathrm{H}), 1.72(1 \mathrm{H}, \mathrm{m}, 1-\mathrm{H}), 1.73(1 \mathrm{H}, \mathrm{m}, 1 \mathrm{a}-\mathrm{H}), 1.75(1 \mathrm{H}, \mathrm{m}, 6-\mathrm{H})$, $1.87(1 \mathrm{H}, \mathrm{dbrd}, J=13.6,2.0 \mathrm{~Hz}, 5-\mathrm{H}), 2.00(1 \mathrm{H}, \mathrm{d}, J=12.8 \mathrm{~Hz}, 4-\mathrm{H}), 2.35$ $\left(3 \mathrm{H}, \mathrm{s}, 2-\mathrm{CH}_{3}\right), 3.03(1 \mathrm{H}, \mathrm{d}, J=12.8 \mathrm{~Hz}, 4-\mathrm{H}), 3.59\left(3 \mathrm{H}, \mathrm{s}, 3-\mathrm{OCH}_{3}\right) .{ }^{13} \mathrm{C}-$ NMR $\left(100 \mathrm{MHz}, \mathrm{CDCl}_{3}\right) \delta: 17.0(\mathrm{C}-1), 18.5(\mathrm{C}-6), 26.8\left(4 \mathrm{a}-\mathrm{CH}_{3}\right), 29.1(8-$ $\left.\mathrm{CH}_{3}\right), 29.9\left(8-\mathrm{CH}_{3}\right), 32.5\left(2-\mathrm{CH}_{3}\right), 32.7(\mathrm{C}-1 \mathrm{a}), 36.0(\mathrm{C}-8), 38.2(\mathrm{C}-4 \mathrm{a}), 40.0$ (C-5), 40.8 (C-7), 43.7 (C-4), 48.0 (C-8a), $51.1\left(3-\mathrm{OCH}_{3}\right), 172.0$ (C-3), 207.4 (C-2). HR-MS (EI) $m / z$ : 266.1870 (Calcd for $\mathrm{C}_{16} \mathrm{H}_{26} \mathrm{O}_{3}: 266.1882$ ).

Acknowledgments We are indebted to Professor Haruki Niwa, Department of Applied Physics and Chemistry, The University of Electro-Communication, for his suggestion on the ruthenium oxidation. We are also grateful to Takasago International Corporation for its kind donation of thujopsene.

\section{References}

1) Yasumoto M., Mada K., Ooi T., Kusumi T., J. Nat. Prod., 63, 1534 1536 (2000).

2) Ohtani I., Kusumi T., Kashman Y., Kakisawa H., J. Am. Chem. Soc., 113, 4092- 4096 (1991).

3) Hasegawa T., Niwa H., Yamada K., Chem. Lett., 1985, 1385-1386.

4) Coudret J. L., Zöllner S., Ravoo B. J., Malara L., Hanisch C., Dörre K., de Meijere A., Waegell B., Tetrahedron Lett., 37, 2425-2428 (1996).

5) Takimoto S., Inanaga J., Katsuki T., Yamaguchi M., Bull. Chem. Soc. Jpn., 54, 1470-1473 (1981).

6) Silverstein R. M., Webster F. X., "Spectrometric Identification of Organic Compounds," 6th edition, John Wiley \& Sons, New York, 1998, p. 191.

7) Tenaglia A., Terranova E., Waegell B., J. Org. Chem., 57, 5523-5528 (1992).

8) Coudret J.-L., Waegell B., Inorganica Chimica Acta, 222, 115-122 (1994).

9) Irie T., Suzuki M., Kurosawa E., Masamune T., Tetrahedron Lett., 1966, 1837-1840.

10) Wolfe S., Hasan S. K., Campbell J. R., Chem. Commun., 1970, 14201421.

11) Webster F. X., Rivas-Enterrios J., Silverstein R. M., J. Org. Chem., 52, 689-691 (1987).

12) Cocker W., Shannon P. V. R., Staniland P. A., J. Chem. Soc. (C), 1966, 946-949. 